\title{
Digital Public Health Technologies and Social Media in Global Emergencies
}

\author{
Patty KOSTKOVA 1 \\ UCL Centre for Digital Public Health in Emergencies (dPHE) \\ UCL, London, United Kingdom
}

Keywords. Digital Health, Emergencies prevention, Community Surveillance, Gamification

In this keynote presentation we will outline the issues surrounding the dissemination of medical evidence and understanding public information needs from Internet searches. Educational effectiveness of serious games highlights the opportunity mobile technology brought to training, community engagement and behaviour change. Social networking with increasing amount of user-generated content from social media and participatory surveillance systems provide readily available source of real-time monitoring and epidemic intelligence to prevent global emergencies such as COVID-19.

Drawing from award winning initiatives we will demonstrate the opportunity for crowdsourcing for community surveillance to combat the zika virus in Brazil, social media use for vaccination campaigns, early warning for epidemics and support for citizens in COVID-19 lockdown through a journaling app, serious mobile games to increasing resilience in perinatal women in Nepal, and decision support tools for antimicrobial prescribing in Nigeria.

\footnotetext{
${ }^{1}$ Corresponding Author: Patty Kostkova, p.kostkova@ucl.ac.uk
} 